\title{
Finitely Additive and Measurable Stochastic Games
}

by

A. Maitra ${ }^{*}$ and W. Sudderth ${ }^{* *}$

University of Minnesota

Technical Report No. 570

January 1992

\begin{abstract}
${ }^{*}$ Research done under the auspices of the Santa Fe Institute Economics Research Program which includes grants from Citicorp/Citibank, Research Corp., the Alex C. Walker Foundation and the Russell Sage Foundation and by grants to SFI from the John D. and Catherine T. MacArthur Foundation, the National Science Foundation (PHY-8714918), and the U.S. Department of Energy (ER-FG05-88ER25054).
\end{abstract}

${ }^{* *}$ Research supported by National Science Foundation Grant DMS-8911548. 


\begin{abstract}
We consider two-person zero-sum stochastic games with arbitrary state and action spaces, a finitely additive law of motion and limit superior payoff function. The players use finitely additive strategies and it is shown that such a game has a value, if the payoff function is evaluated in accordance with the theory of strategic measures as developed by Dubins and Savage. Moreover, when a Borel structure is imposed on the problem, together with an equicontinuity condition on the law of motion, the value of the game is the same whether calculated in terms of countably additive strategies or finitely additive ones.
\end{abstract}

AMS 1980 subject classification: Primary 90D15, Secondary 60G40.

Key words and phrases: two-person zero sum stochastic games, finitely additive strategies, gambling theory, Borel measurable. 


\section{Introduction}

Every two-person, zero-sum, one day game with a bounded payoff function has a value if the players are allowed to use randomized strategies which are finitely additive and if the order of integration is fixed. (This follows from Ky Fan [4, Theorem 2]. A direct proof of the result can be found in Heath and Sudderth [5].) We prove in Theorem 2.1 that the same result holds for stochastic games with arbitrary state and action spaces and a law of motion which corresponds to a finitely additive conditional distribution. The payoff function we study is sufficiently general to include the classical ones such as the long run average reward and the total discounted reward. As far as we are aware, stochastic games in which the law of motion is finitely additive and where the players are allowed to use randomized strategies that are finitely additive have not been studied before, even in the case of the total discounted reward.

The model of stochastic games we consider here was studied in the countably additive setting in [6] and [7]. In [7], it was shown that Borel measurable, countably additive stochastic games have values under appropriate measurability and topological assumptions. In these Borel games players are required to use measurable, countably additive strategies and it is natural to ask if the value would be the same if they were allowed to use finitely additive strategies. As shown in Corollary 9.3, the value is the same under a condition of equicontinuity on the law of motion.

Our study of finitely additive stochastic games and Borel stochastic games is based on analogous work in the Dubins and Savage theory of gambling [1].

\section{Formulation of the problem in the finitely additive setting}

Let $\mathrm{X}, \mathrm{A}, \mathrm{B}$ be nonempty sets. Let $\mathrm{u}$ be a bounded, real-valued function on $\mathrm{X}$ and $\mathrm{q}$ a function which assigns to each triple $(\mathrm{x}, \mathrm{a}, \mathrm{b}) \in \mathrm{X} \times \mathrm{A} \times \mathrm{B}$ a finitely additive probability measure defined on the power-set of $X$. The game $n(u)(x)$ starts at some initial state $X$. Player I chooses an action $a_{1} \in A$ and, simultaneously, player II chooses $b_{1} \in B$. (The players may choose their actions at random, which means that the players choose their actions according to finitely additive probability distributions defined on the power-sets of their respective action sets.) The next state $\mathrm{x}_{1}$ has distribution $\mathrm{q}\left(\cdot \mid \mathrm{x}, \mathrm{a}_{1}, \mathrm{~b}_{1}\right)$ and is announced to the players along with their chosen actions. The procedure is iterated so as to generate a random sequence $x_{1}, x_{2}, \ldots$ and the payoff from $I I$ to $I$ is

$$
u^{*}=\lim _{n} \sup u\left(x_{n}\right)
$$


We will next indicate how the distribution of the random sequence $x_{1}, x_{2}, \ldots$ is defined in the finitely additive theory.

A strategy $\sigma$ for player $I$ is a sequence $\sigma_{0}, \sigma_{1}, \sigma_{2}, \ldots$ such that $\sigma_{0} \in \mathrm{P}(\mathrm{A})$ and, for $n \geq 1, \sigma_{n}$ is a mapping from $Z^{n}$ into $P(A)$, where $Z=A \times B \times X$ and $P(A)$ is the set of all finitely additive probability measures on the power-set of $A$. One defines a strategy $\tau$ for player II in an analogous manner, with $\mathrm{P}(\mathrm{A})$ replaced by $\mathrm{P}(\mathrm{B})$, the set of finitely additive probability measures on the power-set of $B$.

Associated with the stochastic games $n(u)(x), x \in X$, is a gambling house, in the sense of Dubins and Savage [1], defined as follows.

Define the state space to be $Y=X \cup Z$. For each triple $(x, \mu, v) \in X \times P(A) \times$ $P(B)$, let $m(x, \mu, v)$ be the finitely additive probability measure on the power-set of $Z$ defined by

$$
m(x, \mu, v)(F)=\iint q\left(F_{a, b} \mid x, a, b\right) d \mu(a) d v(b),
$$

where $\mathrm{F} \subseteq \mathrm{Z}$ and $\mathrm{F}_{\mathrm{a}, \mathrm{b}}=\{\mathrm{x} \in \mathrm{X}:(\mathrm{a}, \mathrm{b}, \mathrm{x}) \in \mathrm{F}\}$. Here the order of integration is important and throughout this article we will integrate in the order adopted above. We can now complete the definition of the gambling house. Set

$$
\Gamma(x)=\{m(x, \mu, v): \mu \in P(A), v \in P(B)\}, x \in X,
$$

and

$$
\Gamma(\mathrm{z})=\{\mathrm{m}(\mathrm{x}, \mu, v): \mu \in \mathrm{P}(\mathrm{A}), v \in \mathrm{P}(\mathrm{B})\}
$$

where $\mathrm{z}=(\mathrm{a}, \mathrm{b}, \mathrm{x}) \in \mathrm{Z}$.

Let $\sigma, \tau$ be strategies for players I and II, respectively, in the game $n(u)(x)$. Then $\sigma$ and $\tau$ induce a strategy $<\sigma, \tau>$ in the gambling house $\Gamma$ as follows:

$$
<\sigma, \tau>_{0}=m\left(x, \sigma_{0}, \tau_{0}\right)
$$

and, for $\mathrm{n} \geq 1$, 


$$
\begin{aligned}
\left\langle\sigma, \tau>_{n}\left(y_{1}, y_{2}, \ldots, y_{n}\right)\right. & =m\left(x_{n}, \sigma_{n}\left(y_{1}, y_{2}, \ldots, y_{n}\right), \tau_{n}\left(y_{1}, y_{2}, \ldots, y_{n}\right)\right) \\
& \text { if }\left(y_{1}, \ldots, y_{n}\right) \in Z^{n} \text { and } y_{n}=\left(a_{n}, b_{n}, x_{n}\right) \\
& =m\left(x_{n}, \sigma_{0}, \tau_{0}\right) \text { if }\left(y_{1}, y_{2}, \ldots, y_{n}\right) \notin Z^{n} \text { and } y_{n}=\left(a_{n}, b_{n}, x_{n}\right) \text { or } x_{n} .
\end{aligned}
$$

Then $\langle\sigma, \tau\rangle$ is a strategy available at $\mathrm{x} \in \mathrm{X}$ in the gambling house $\Gamma$ and therefore induces a finitely additive probability measure $P_{x, \sigma, \tau}$ on the Borel subsets of $Y^{N}$, where $N=\{1,2, \ldots\}$ and $Y^{N}$ is endowed with the product of copies of the discrete topology on $Y$. The probability measure $P_{x, \sigma, \tau}$ was defined on the field of clopen sets by Dubins and Savage [1] and then extended to the Borel $\sigma$-field by Dubins [2] and Purves and Sudderth [10]. In the sequel we shall freely use the properties established for $P_{x, \sigma, \tau}$ by DubinsSavage-Purves-Sudderth in [1], [2] and [10]. Note that $P_{x, \sigma, \tau}\left(Z^{N}\right)=1$.

To return to the game $n(u)(x)$, we can now define the expected payoff that accrues to player I from player II, when I uses $\sigma$ and II uses $\tau$, as

$$
E_{x, \sigma, \tau}\left(u^{*}\right)=\int u^{*} d P_{x, \sigma, \tau} .
$$

In the sequel, we will sometimes drop $\mathrm{x}$, especially when it is clear from the context, and write $P_{\sigma, \tau}$ and $E_{\sigma, \tau}$ for $P_{x, \sigma, \tau}$ and $E_{x, \sigma, \tau}$, respectively.

The upper and lower values $\bar{V}(x), \underline{V}(x)$ of the game $n(u)(x)$ can be defined in the usual manner:

$$
\bar{V}(x)=\inf _{\tau} \sup _{\sigma} E_{x, \sigma, \tau}\left(u^{*}\right)
$$

and

$$
\underline{V}(x)=\sup _{\sigma} \inf _{\tau} E_{x, \sigma, \tau}\left(u^{*}\right),
$$

where the sup is over all strategies $\sigma$ of player I and the inf is over all strategies $\tau$ of player II.

Theorem 2.1. For every $x \in X$, the game $n(u)(x)$ has a value, that is,

$$
\bar{V}(x)=\underline{V}(x) .
$$




\section{Notation.}

Let $\mathrm{Z}=\mathrm{A} \times \mathrm{B} \times \mathrm{X}$ and define the space of histories to be $\mathrm{H}=\mathrm{Z}^{\mathrm{N}}=\mathrm{Z} \times \mathrm{Z} \times \ldots$. Elements of $H$ will be denoted by $h=\left(z_{1}, z_{2}, \ldots\right)$. We use $p_{n}(h)$, or more briefly, $p_{n}$ to denote the partial history $\left(\mathrm{z}_{1}, \mathrm{z}_{2}, \ldots, \mathrm{z}_{\mathrm{n}}\right)$.

Suppose $\sigma$ is a strategy for player $I$ in the stochastic game and $p=\left(z_{1}, z_{2}, \ldots, z_{n}\right)$ a partial history. Then the conditional strategy $\sigma[p]$ is defined by

$$
\begin{gathered}
\sigma[\mathrm{p}]_{0}=\sigma_{\mathrm{n}}(\mathrm{p}) \\
\sigma[\mathrm{p}]_{\mathrm{m}}\left(\mathrm{z}_{1}^{\prime}, \mathrm{z}_{2}^{\prime}, \ldots, \mathrm{z}_{\mathrm{m}}^{\prime}\right)=\sigma_{\mathrm{n}+\mathrm{m}^{\prime}}\left(\mathrm{z}_{1}, \mathrm{z}_{2}, \ldots, \mathrm{z}_{\mathrm{n}}, \mathrm{z}_{1}^{\prime}, \mathrm{z}_{2}^{\prime}, \ldots, \mathrm{z}_{\mathrm{m}}^{\prime}\right)
\end{gathered}
$$

for all $m \geq 1$ and $\left(z_{1}^{\prime}, z_{2}^{\prime}, \ldots, z_{m}^{\prime}\right) \in Z^{m}$. If $\tau$ is a strategy for player $I$, the definition of the conditional strategy $\tau[\mathrm{p}]$ is similar. Given strategies $\sigma$ and $\tau$ for players I and II, an initial state $\mathrm{x} \in \mathrm{X}$ and a bounded, Borel measurable function $\mathrm{g:} \mathrm{H} \rightarrow \Re$, we have:

$$
E_{\sigma, \tau}(g)=\int\left[E_{\sigma\left[p_{n}(h)\right], \tau\left[p_{n}(h)\right]}\left(g p_{n}(h)\right\} d P_{\sigma, \tau}(h),\right.
$$

where, for $\mathrm{p}=\mathrm{p}_{\mathrm{n}}(\mathrm{h})=\left(\mathrm{z}_{1}, \mathrm{z}_{2}, \ldots, \mathrm{z}_{\mathrm{n}}\right)$, gp is the $\mathrm{p}$-section of $\mathrm{g}$ defined on $\mathrm{H}$ by $\mathrm{gp}\left(\mathrm{h}^{\prime}\right)=$ $g p\left(z_{1}^{\prime}, z_{2}^{\prime}, \ldots\right)=g\left(z_{1}, z_{2}, \ldots, z_{n}, z_{1}^{\prime}, z_{2}^{\prime}, \ldots\right)$. In the special case when $g(h)=u^{*}(h)=$ $\lim _{n} \sup u\left(x_{n}\right), u^{*} p$ is just $u^{*}$ and (3.1) simplifies to

$$
E_{\sigma, \tau}\left(u^{*}\right)=\int\left\{E_{\sigma\left[p_{n}(h)\right], \tau\left[p_{n}(h)\right]}\left(u^{*}\right)\right\} d P_{\sigma, \tau}(h) .
$$

In the conventional, countably additive theory, formula (3.1) would be an instance of the familiar fact that the expectation of a random variable can be written as the expectation of its conditional expectation given another random variable or sigma-field. In the finitely additive theory of Dubins and Savage, it was proved for finitary (continuous) $g$ in [1] and, more generally, for bounded, Borel $g$ in [10].

A stopping time $t$ is a mapping from $H$ to $\{0,1, \ldots\} \cup\{\infty\}$ such that, for $n=0,1, \ldots$, if $t(h)=n$ and $h^{\prime}$ agrees with $h$ in the first $n$ coordinates, then $t\left(h^{\prime}\right)=n$. Note that if $t(h)=0$ for some $h$, then $t$ is identically zero. A stop rule is a stopping time which is everywhere finite. 
If $t$ is a stopping time, $h=\left(z_{1}, z_{2}, \ldots\right)=\left(\left(a_{1}, b_{1}, x_{1}\right),\left(a_{2}, b_{2}, x_{2}\right), \ldots\right)$, and $t(h)<\infty$, we define the variables $z_{t}, x_{t}, p_{t}$ to have values $z_{t(h)}, x_{t(h)}, p_{t}(h)=\left(z_{1}, z_{2}, \ldots, z_{t}(h)\right.$ at $h$. For strategies $\sigma$ and $\tau$ of players I and II, formula (3.1) generalizes to

$$
E_{\sigma, \tau}(g)=\int\left\{E_{\sigma\left[p_{\mathfrak{l}}, \tau\left[p_{\mathfrak{l}}\right]\right.}\left(g p_{\mathfrak{l}}\right)\right] d P_{\sigma, \tau}
$$

Like formula (3.1), formula (3.2) was proved for finitary $g$ in [1] and then extended to bounded, Borel $\mathrm{g}$ in [11].

If $t$ is a stop rule and $p=\left(z_{1}, z_{2}, \ldots, z_{n}\right)$ a partial history, define $t[p]$ on $H$ by

$$
t[p]\left(z_{1}^{\prime}, z_{2}^{\prime}, \ldots\right)=t\left(z_{1}, z_{2}, \ldots, z_{n}, z_{1}^{\prime}, z_{2}^{\prime}, \ldots\right)-n
$$

Notice that, if $t\left(z_{1}, z_{2}, \ldots, z_{n}, \ldots\right) \geq n$, then $t[p]$ is itself a stop rule, in which case $t[p]$ is called a conditional stop rule given $p$. When $p=(z)$, we write $z$ for $p$ and $t[z]$ for $t[p]$.

There is a natural way to associate with every stop rule $t$ an ordinal number $j(t)$ called the index of $t$ by setting $j(0)=0$ and requiring, for $t>0$, that

$$
j(t)=\sup (j(t[z])+1: z \in Z\}
$$

The ordinal function $j(t)$ is familiar to students of Dubins and Savage as being the structure of the finitary function $z_{l}([1$,sections 2.7 and 2.9]) except for the uninteresting case when $Z$ is a singleton. Some of our proofs will use transfinite induction on $j(t)$ and it is important to notice that, for all $t>0$ and all $z \in Z, j(t[z])$ is strictly less than $j(t)$.

Suppose $t>0$ is a stop rule and consider the special case of (3.1) when $n=1$ and $g=u\left(x_{t}\right)$. Note that

$$
\left(x_{t} z_{1}\right)\left(z_{2}, z_{3}, \ldots\right)=x_{t}\left(z_{1}, z_{2}, \ldots\right)=x_{t\left[z_{1}\right]}\left(z_{2}, z_{3}, \ldots\right)
$$

if we make the convention that $x_{t\left[z_{1}\right]}\left(z_{2}, z_{3}, \ldots\right)=x_{1}$ when $t\left[z_{1}\right]=0$. Then (3.1) yields

$$
E_{\sigma, \tau}\left(u\left(x_{t}\right)\right)=\int\left\{E_{\sigma\left[z_{1}\right], \tau\left[z_{1}\right]}\left(u\left(x_{t}\left[z_{1}\right]\right)\right)\right\} d P_{\sigma_{0, \tau}}\left(z_{1}\right) .
$$




\section{Auxiliary games}

Consider an auxiliary one-day game $Q(\varphi)(x)$ starting from $x$, where $\varphi$ is a bounded function on $X$. In the game $Q(\varphi)(x)$, players I and II choose actions a,b simultaneously with $a \in A$ and $b \in B$ and the payoff from II to $I$ is

$$
\int \varphi\left(x_{1}\right) q\left(d x_{1} \mid x, a, b\right) .
$$

The expected payoff from II to I when I plays a random strategy $\mu \in \mathrm{P}(\mathrm{A})$ and II plays $v \in$ $\mathrm{P}(\mathrm{B})$ is

$$
\iiint \varphi\left(x_{1}\right) q\left(d x_{1} \mid x, a, b\right) d \mu(a) d v(b),
$$

which we abbreviate by $\tilde{\varphi}(\mu, v)$. Again the order of integration is important and is consistent with the order of integration adopted in section 2 . Note that $\tilde{\varphi}(\mu, v)$ is just the expectation of $\varphi$ with respect to the probability measure $m(x, \mu, v)$.

Lemma 4.1. For any bounded function $\varphi$ on $X$ and any $x \in X$, the game $Q(\varphi)(x)$ has value $(S \varphi)(x)$, that is,

$$
(S \varphi)(x)=\inf _{v \in P(B)} \sup _{\mu \in P(A)} \tilde{\varphi}(\mu, v)=\sup _{\mu \in P(A)} \inf _{v \in P(B)} \tilde{\varphi}(\mu, v) .
$$

Moreover, both players have optimal strategies, which may be random.

Lemma 4.1 is an easy consequence of Theorem 2 in Ky Fan [4].

Next, we define functions $U_{\xi}$ by transfinite induction on $\xi$ as follows:

$$
\mathrm{U}_{0}=\mathrm{u}
$$

and, for $\xi>0$,

$$
\begin{array}{rlrl}
U_{\xi} & =S U_{\eta} \vee u & & \text { if } \xi=\eta+1 \\
& =\sup _{\eta<\xi} U_{\eta} & \text { if } \xi \text { is limit, }
\end{array}
$$


where $a \vee b$ is the maximum of $a$ and $b$. Let

$$
\mathrm{U}=\sup _{\xi} U_{\xi}
$$

Lemma 4.2. $U$ is the least, bounded function $\varphi$ on $X$ such that (a) $\varphi \geq u$ and (b) $\varphi \geq S \varphi$.

Proof. First, note that the operator $S$ is monotone. So, if $\varphi$ satisfies (a) and (b), then, by transfinite induction on $\xi, \varphi \geq U_{\xi}$ for every $\xi$. Plainly, $U \geq u$. Moreover, $U_{\xi} \geq U_{\eta}$ if $\eta<$ $\xi$. So there is $\xi^{*}$ such that $U_{\xi^{*}}=U_{\xi^{*}+1}$, as can be seen by using an easy cardinality argument. It follows that $U=U_{\xi *}$. Hence,

$$
\mathrm{SU}=\mathrm{SU}_{\xi^{*}} \leq \mathrm{U}_{\xi^{*}+1}=\mathrm{U} \text {. }
$$

Lemma 4.3. $U=u \vee S U$.

Proof. Choose an ordinal $\xi^{*}$ such that $U_{\xi^{*}}=U_{\xi^{*}+1}$. It follows that $U=U_{\xi^{*}}$. Hence

$$
U=U_{\xi^{*}+1}=u \vee S U_{\xi^{*}}=u \vee S U .
$$

Now consider a game $\mathscr{L}(u)(x)$, determined by an initial position $x$ and the utility function $u$ and played exactly.like the game $n(u)(x)$ of section 2 , except that now player I gets to terminate the game unilaterally at any time of his choice and the payoff to I from II is the value of $u$ at the terminal state. More formally, I chooses a strategy $\sigma$ and a stop rule $t$, II chooses a strategy $\tau$ and the expected payoff to I from II is $E_{x, \sigma, \tau}\left(u\left(x_{t}\right)\right)$. Here we allow $\mathrm{t}=0$ and require $\mathrm{x}_{0}=\mathrm{x}$.

The next three lemmas will prove that the game $\mathscr{L}(u)(x)$ has value $U(x)$.

Lemma 4.4. For every $x \in X$, ordinal $\xi \geq 0$ and $\varepsilon>0$, I has a strategy $\sigma_{\varepsilon}^{\xi}(x)$ and a stop rule ${\underset{\varepsilon}{\xi}}_{\varepsilon}^{\xi}(x)$ such that for any strategy $\tau$ of II,

$$
{ }_{\sigma_{\varepsilon}^{\xi}(x), \tau}\left(u\left(x_{\xi}\right)\right) \geq U_{\xi}(x)-\varepsilon
$$

for every $x \in X$. 
Proof. Fix $\mu^{*} \in P(A)$. For $\xi=0$, let $t_{\varepsilon}^{0}(x) \equiv 0$ and $\sigma_{\varepsilon}^{0}(x)$ be the strategy which uses $\mu^{*}$ every day.

Assume the result is true for all $\eta<\xi$. Let $\xi$ be a limit ordinal. Set

$$
\begin{aligned}
\mathfrak{t}_{\varepsilon}^{\xi}(x)(h) & =0 & & \text { if } u(x) \geq U_{\xi}(x) \\
& =t_{\varepsilon / 2}^{\eta(x)}(h) & & \text { if } u(x)<U_{\xi}(x),
\end{aligned}
$$

where $\eta(x)$ is the least $\eta$ such that $U_{\eta}(x) \geq U_{\xi}(x)-\varepsilon / 2$; and

$$
\begin{aligned}
\sigma_{\varepsilon}^{\xi}(x) & =\sigma_{\varepsilon}^{0}(x) \quad \text { if } u(x) \geq U_{\xi}(x) \\
& =\sigma_{\varepsilon / 2}^{\eta(x)}(x) \quad \text { if } u(x)<U_{\xi}(x) .
\end{aligned}
$$

It is easy to verify that $\sigma_{\varepsilon}^{\xi}(x)$ and $t_{\varepsilon}^{\xi}(x)$ satisfy (4.1).

Suppose next that $\xi=\eta+1$. For each $x \in X$, let $f(x) \in P(A)$ be optimal for $I$ in the game $Q\left(U_{\eta}\right)(x)$. Consider as above two cases.

Case 1. $\quad u(x) \geq U_{\xi}(x)$. Then define

$$
\sigma_{\varepsilon}^{\xi}(x)=\sigma_{\varepsilon}^{0}(x)
$$

and

$$
t_{\varepsilon}^{\xi}(x)=t_{\varepsilon}^{0}(x)
$$

Now (4.1) is obvious.

Case 2. $\quad u(x)<U_{\xi}(x)$. Then set

$$
\begin{aligned}
& \sigma_{\varepsilon}^{\xi}(x)_{0}=f(x) \\
& \sigma_{\varepsilon}^{\xi}(x)_{n}\left(z_{1}, z_{2}, \ldots, z_{n}\right)=\sigma_{\varepsilon}^{\eta}\left(x_{1}\right)_{n-1}\left(z_{2}, z_{3}, \ldots, z_{n}\right), n \geq 1, \\
& t_{\varepsilon}^{\xi}(x)(h)=t_{\varepsilon}^{\eta}\left(x_{1}\right)\left(z_{2}, z_{3}, \ldots\right)+1 .
\end{aligned}
$$


So, if $\tau$ is any strategy of player II, then

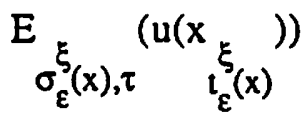

$$
\begin{aligned}
& =\iint u\left(x_{\mathfrak{t}_{\varepsilon}(x)\left[z_{1}\right]}\right) d P \underset{\sigma_{\varepsilon}^{\xi}(x)\left[z_{1}\right], \tau\left[z_{1}\right]}{d P} \quad \sigma_{\varepsilon}^{\xi}(x), \tau \\
& =\iiint_{t_{\varepsilon}^{\prime}\left(x_{1}\right)}^{u\left(x_{\eta}\right)} d P \underset{\sigma_{\varepsilon}^{\eta}\left(x_{1}\right), \tau\left[z_{1}\right]}{d P} \sigma_{\varepsilon}^{\xi}(x), \tau \\
& \geq \iiint U_{\eta}\left(x_{1}\right) q\left(d x_{1} \mid x, a, b\right) f(x)(d a) \tau_{0}(d b)-\varepsilon \\
& \geq\left(\mathrm{SU}_{\eta}\right)(\mathrm{x})-\varepsilon \\
& =U_{\xi}(x)-\varepsilon .
\end{aligned}
$$

Lemma 4.5. For every $x \in X$ and $\varepsilon>0$, player I has a strategy $\sigma_{\varepsilon}(x)$ and a stop rule $t_{\varepsilon}(x)$ such that for any $\tau$ played by $\Pi$,

$$
E_{\sigma_{\varepsilon}(x), \tau}\left(u\left(x_{l_{\varepsilon}}(x)\right)\right) \geq U(x)-\varepsilon
$$

for every $x \in X$.

Proof. Choose an ordinal $\xi^{*}$ such that $U=U_{\xi^{*}}$. Define

$$
\sigma_{\varepsilon}(x)=\sigma_{\varepsilon}^{\xi^{*}}(x), x \in X,
$$

and

$$
t_{\varepsilon}(x)=t_{\varepsilon}^{\xi^{*}}(x), x \in X
$$

where $\sigma_{\varepsilon}^{\xi^{*}}(x)$ and $\xi_{\varepsilon}^{\xi^{*}}(x)$ are as in Lemma 4.4. Lemma 4.5 follows immediately from Lemma 4.4. 
Lemma 4.6. For every $x \in X$, player II has a strategy $\tau(x)$ such that for any strategy $\sigma$ of $I$ and any stop rule $t$,

$$
\mathrm{E}_{\sigma, \tau(\mathrm{x})}\left(\mathrm{u}\left(\mathrm{x}_{\mathrm{t}}\right)\right) \leq \mathrm{U}(\mathrm{x})
$$

for every $x \in X$.

Proof. For every $x \in X$, let $g(x) \in P(B)$ be optimal for II in the game $Q(U)(x)$. Define

$$
\tau(\mathrm{x})_{0}=\mathrm{g}(\mathrm{x})
$$

and

$$
\tau(x)_{n}\left(z_{1}, z_{2}, \ldots, z_{n}\right)=g\left(x_{n}\right), n \geq 1
$$

Suppose now that $\sigma$ is a strategy of $I$ and $t$ a stop rule. We will prove (4.3) by induction on $j(t)$. For $t \equiv 0,(4.3)$ is obvious. For the inductive step, calculate as follows:

$$
\begin{aligned}
E_{\sigma, \tau(x)}\left(u\left(x_{t}\right)\right) & =\iint u\left(x_{t\left[z_{1}\right]}\right) d P_{\sigma\left[z_{1}\right], \tau(x)\left[z_{1}\right]} d P_{\sigma, \tau(x)} \\
& =\iint u\left(x_{t\left[z_{1}\right]}\right) d P_{\sigma\left[z_{1}\right], \tau\left(x_{1}\right)} d P_{\sigma, \tau(x)} \\
& \leq \int U\left(x_{1}\right) d P_{\sigma, \tau(x)} \\
& =\iiint U\left(x_{1}\right) q\left(d x_{1} \mid x, a, b\right) \sigma_{0}(d a) g(x)(d b) \\
& \leq(S U)(x) \\
& \leq U(x),
\end{aligned}
$$

where the first inequality is by virtue of the inductive hypothesis and the last one is by virtue of Lemma 4.2.

Combining Lemmas 4.5 and 4.6, we get:

Theorem 4.7. For every $x \in X$, the game $\mathcal{L}(u)(x)$ has value $U(x)$.

As the final auxiliary game, we consider a modification $2^{*}(u)(x)$ of the game $\mathcal{L}(u)(x)$. In the game $\mathcal{L}^{*}(u)(x)$, player I chooses a strategy $\sigma$ and a stop rule $t \geq 1$, player II chooses a strategy $\tau$ and the expected payoff from II to I is $E_{x, \sigma, \tau}\left(u\left(x_{t}\right)\right)$. The only difference between the games $\mathcal{L}^{*}(u)(x)$ and $\mathscr{L}(u)(x)$ is that in the former, I is not allowed to choose $t=0$. 
Theorem 4.8. For every $x \in X$ and every $\varepsilon>0$, I has a strategy $\sigma_{\varepsilon}(x)$ and a stop rule $t_{\varepsilon}(x)$ $\geq 1$ such that for any strategy $\tau$ of $I$,

$$
E_{\sigma_{\varepsilon}(x) \tau}\left(u\left(x_{t_{\varepsilon}(x)}\right)\right) \geq(S U)(x)-\varepsilon \text {. }
$$

Moreover, for every $x \in X$, II has a strategy $\tau(x)$ such that for any strategy $\sigma$ of $I$ and any stop rule $\mathrm{t} \geq 1$,

$$
E_{\sigma, \tau(x)}\left(u\left(x_{t}\right)\right) \leq(S U)(x)
$$

Lastly, for every $x \in X$, the game $\mathfrak{L}^{*}(u)(x)$ has value (SU)(x).

Proof. Let $\bar{X}$ be a copy of $X$ and disjoint with $X$. If $X \in X$, its copy in $\bar{X}$ will be denoted by $\bar{x}$. Consider a new problem with state space $X \cup \bar{X}$, the same action sets $A, B$ as in the original problem, the same utility $u$ and the same law of motion $q$ on $X$ and extended to $\bar{X}$ as follows,

$$
u(\bar{x})=\inf \{u(y): y \in X\}-1
$$

and

$$
q(\cdot \bar{x}, a, b)=q(\cdot \mid x, a, b)
$$

Note that, for any $x \in X$, the game $\mathscr{L}(u)(\bar{x})$ is equivalent to $\mathcal{L}^{*}(u)(x)$ because I will not have any incentive to use $t=0$ if the starting state is $\bar{x}$. Consequently, $U(\bar{x})$, the value of the game $\mathcal{L}(\mathrm{u})(\overline{\mathrm{x}})$, will also be value of the game $\mathcal{L}^{*}(\mathrm{u})(\mathrm{x})$. By Lemma 4.3,

$$
U(\bar{x})=u(\bar{x}) \vee(S U)(\bar{x})=(S U)(\bar{x})=(S U)(x)
$$

Hence the value of $2^{*}(u)(x)$ is $(S U)(x)$. The remaining assertions follow by applying Lemma 4.5 and Lemma 4.6 to the games $\mathcal{L}(u)(\bar{x}), \bar{x} \in \bar{X}$, and by recalling from the proof of Lemma 4.6 that for any $x \in X$, any strategy $\sigma$ of $I$ and any stop rule $t \geq 1$,

$$
\mathrm{E}_{\sigma, \tau(\mathrm{x})}\left(\mathrm{u}\left(\mathrm{x}_{\mathrm{t}}\right)\right) \leq \mathrm{SU}(\mathrm{x})
$$

We now define a new operator $\mathrm{T}$ by setting for any bounded, real-valued function w on X 


$$
(T w)(x)=\text { value of the game } Z^{*}(w)(x), x \in X
$$

\section{Proof of Theorem 2.1.}

We now return to the games $n(\mathrm{u})(\mathrm{x})$. We will need for the proof of Theorem 2.1 a preliminary result from the Dubins-Savage theory of gambling, which will enable us to approximate the game $n(u)(x)$ by the game $2^{*}(u)(x)$.

Lemma 5.1. Let $\sigma, \tau$ be strategies for players I and II, respectively. Then, for any $x \in X$,

$$
\int u^{*} d P_{x, \sigma, \tau}=\inf _{s} \sup _{t \geq s} \int u\left(x_{t}\right) d P_{x, \sigma, \tau},
$$

where $s, t$ run over stop rules which are $\geq 1$.

Formula (5.1) is called the Fatou equation and was proved in the countably additive setting by Sudderth [12] and then extended to the finitely additive setting by Purves and Sudderth [9, Theorem 9.4].

Theorem 5.2. Suppose $\varphi$ is a bounded, real-valued function on $X$ such that $T(u \wedge \varphi) \geq \varphi$. Then $\underline{\mathrm{V}} \geq \varphi$.

Proof. Let $\varepsilon>0$. Choose $\delta_{n}, n \geq 0$, such that $\delta_{n}>0$ and $\sum_{0}^{\infty} \delta_{n}<\varepsilon$. By Theorem 4.8, for each $x \in X$ and $n \geq 0$, I has a strategy $\sigma^{n}(x)$ and a stop rule $t_{n}(x) \geq 1$ such that for any strategy $\tau$ of $\mathrm{II}$,

$$
\begin{aligned}
E_{\sigma^{n}(x), \tau}\left(u \wedge \varphi\left(x_{L_{n}(x)}\right)\right) & \geq(T(u \wedge \varphi))(x)-\delta_{n} \\
& \geq \varphi(x)-\delta_{n} .
\end{aligned}
$$

Let $\sigma(x)$ be the sequential composition of $\left(\sigma^{n}, t_{n}\right), n \geq 0$, at $x$ as defined in Dubins et al. [3]. In detail, first define stop rules $1 \leq s_{0}(x)<s_{1}(x)<\ldots$ by setting for $h=\left(z_{1}, z_{2}, \ldots\right)$,

$$
s_{0}(x)(h)=t_{0}(x)(h)
$$

and 


$$
s_{n+1}(x)(h)=s_{n}(x)(h)+t_{n+1}\left(z_{s_{n}(x)}\right)\left(z_{s_{n}(x)+1}, z_{\left.s_{n}(x)+2, \ldots\right)}\right.
$$

[Recall that $z_{t}(h)=z_{t(h)}$ for a stop rule $t$ and $h \in H$.] Now define $\sigma(x)$ as follows:

$$
\begin{aligned}
\sigma(x)_{0} & =\sigma^{0}(x)_{0} \\
\sigma(x)_{n}\left(z_{1}, z_{2}, \ldots, z_{n}\right) & =\sigma^{0}(x)_{n}\left(z_{1}, z_{2}, \ldots, z_{n}\right) \text { if } 1 \leq n<s_{0}(x)(h) \\
& =\sigma^{k+1}\left(z_{s_{k}(x)}\right)_{n-s_{k}(x)}\left(z_{s_{k}}(x)+1, z_{s_{k}}(x)+2, \ldots, z_{n}\right) \\
& \text { if } s_{k}(x)(h) \leq n<s_{k+1}(x)(h)
\end{aligned}
$$

where $h=\left(z_{1}, z_{2}, \ldots, z_{n}, \ldots\right)$. Thus, $\sigma(x)$ follows $\sigma^{0}(x)$ up to time $s_{0}(x)$, then switches to $\sigma^{1}\left(x_{s_{0}}(x)\right.$ and so on.

We claim that for every $x \in X$ and stop rule $s \geq 1$, there is a stop rule $t \geq s$ such that for every strategy $\tau$ of player II,

$$
E_{x, \sigma(x), \tau}\left(u\left(x_{t}\right)\right) \geq \varphi(x)-\sum_{0}^{\infty} \delta_{n} .
$$

The claim will be proved by induction on the index $\mathrm{j}(\mathrm{s})$.

Fix $\mathrm{x}_{0} \in \mathrm{X}$ and write $\sigma$ for $\sigma\left(\mathrm{x}_{0}\right)$. To start the induction, suppose that $s \equiv 1$. Let $\mathrm{t}$ $=t_{0}\left(x_{0}\right)$. Then, for any strategy $\tau$ of II,

$$
\begin{aligned}
\mathrm{E}_{\mathrm{x}_{0}, \sigma, \tau}\left(\mathrm{u}\left(\mathrm{x}_{\mathrm{t}}\right)\right) & =\mathrm{E}_{\sigma 0\left(\mathrm{x}_{0}\right), \tau}\left(\mathrm { u } \left(\mathrm{x}_{\left.\left.\mathrm{t}_{0}\left(\mathrm{x}_{0}\right)\right)\right)}\right.\right. \\
& \geq \mathrm{E}_{\sigma 0\left(\mathrm{x}_{0}\right), \tau}\left(\mathrm { u } \wedge \varphi \left(\mathrm{x}_{\left.\left.\mathrm{t}_{0}\left(\mathrm{x}_{0}\right)\right)\right)}\right.\right. \\
& \geq \varphi\left(\mathrm{x}_{0}\right)-\delta_{0} \\
& \geq \varphi\left(\mathrm{x}_{0}\right)-\sum_{0}^{\infty} \delta_{\mathrm{n}},
\end{aligned}
$$

where the second inequality is by virtue of (5.2).

For the inductive step, assume that $j(s)>1$ and that the claim is true for all stop rules $s^{\prime} \geq 1$ with $j\left(s^{\prime}\right)<j(s)$. Write $t_{0}$ for $t_{0}\left(x_{0}\right)$. 
Fix $h \in H$ and suppose that $s(h)>t_{0}(h)$. Let $\bar{s}_{h}=s\left[p_{t_{0}}(h)\right]$, so that $j\left(\bar{s}_{h}\right)<j(s)$. Set $\bar{\sigma}_{h}=\sigma\left[p_{t_{0}}(h)\right]$, so that $\bar{\sigma}_{h}$ is the sequential composition of $\left(\sigma^{n}, t_{n}\right), n \geq 1$, at $x_{t_{0}}$. Apply the inductive hypothesis to $\bar{\sigma}_{h}, \bar{s}_{h}, x_{L_{0}}$ to get a stop rule $\bar{t}_{h}$ (depending on $h$ only through $\left.\mathrm{p}_{\mathrm{t}_{0}}(\mathrm{~h})\right) \geq \bar{s}_{\mathrm{h}}$ such that for every strategy $\tau^{\prime}$ of II

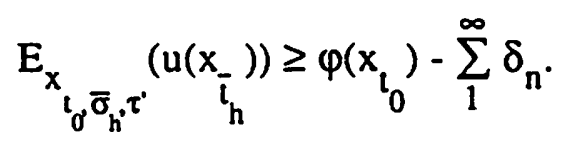

Now define, for any $h \in H$,

$$
\begin{aligned}
t(h) & =t_{0}(h)+\bar{t}_{h}\left(z_{t_{0}+1}, z_{t_{0}+2}, \ldots\right) \text { if } t_{0}(h)<s(h), \\
& =t_{0}(h) \quad \text { if } s(h) \leq t_{0}(h) .
\end{aligned}
$$

Then $t$ is a stop rule and it is straightforward to verify that $t \geq s$. Hence, for any strategy $\tau$ of II,

$$
\begin{aligned}
& E_{x_{0}, \sigma, \tau}\left(u\left(x_{v}\right)\right) \\
& =\int_{\left(s \leq t_{0}\right)} u\left(x_{t}\right) d P_{\sigma, \tau}+\int_{\left(s>t_{0}\right]} u\left(x_{t}\right) d P_{\sigma, \tau} \\
& =\int\left(s \leq t_{0}\right) u\left(x_{t_{0}}\right) d P_{\sigma, \tau}+\int_{\left\{s>t_{0}\right\}} E_{\bar{\sigma}_{h}, \tau\left[p_{t_{0}}(h)\right]}\left(u\left(x_{\bar{t}_{h}}\right)\right) d P_{\sigma, \tau} \\
& \geq \int\left(s \leq t_{0}\right) u\left(x_{t_{0}}\right) d P_{\sigma, \tau}+\int\left(s>t_{0}\right) \varphi\left(x_{t_{0}}\right) d P_{\sigma, \tau}-\sum_{1}^{\infty} \delta_{n} \\
& \geq \int(u \wedge \varphi)\left(x_{L_{0}}\right) d P_{\sigma, \tau}-\sum_{1}^{\infty} \delta_{n} \\
& =\mathrm{E}_{\mathrm{x}_{0}, \sigma^{0}\left(\mathrm{x}_{0}\right), \tau}\left(\mathrm{u} \wedge \varphi\left(\mathrm{x}_{\mathrm{t}_{0}}\right)\right)-\sum_{1}^{\infty} \delta_{\mathrm{n}} \\
& \geq \varphi\left(x_{0}\right)-\sum_{0}^{\infty} \delta_{n},
\end{aligned}
$$


where the first inequality is by virtue of (5.4) and the penultimate equality is by virtue of (5.2).

It now follows from the claim and Lemma 5.1 that for any $x \in X$ and any strategy $\tau$ of II,

$$
\mathrm{E}_{\mathrm{x}, \sigma(\mathrm{x}), \tau}\left(\mathrm{u}^{*}\right) \geq \varphi(\mathrm{x})-\varepsilon
$$

so that

$$
\underline{V}(x) \geq \varphi(x)-\varepsilon \text {. }
$$

Since $\varepsilon$ is arbitrary, this completes the proof.

In order to complete the proof of Theorem 2.1, it now remains to prove

Theorem 5.3. $T(u \wedge \bar{V}) \geq \bar{V}$.

Proof. Fix $x \in X$ and $\varepsilon>0$. Let $\tau^{1}$ be optimal for II in the game $\mathcal{L}^{*}(u \wedge \bar{V})(x)$, that is,

$$
\mathrm{E}_{\sigma, \tau^{1}}\left(\mathrm{u} \wedge \overline{\mathrm{V}}\left(\mathrm{x}_{\mathfrak{t}}\right)\right) \leq(\mathrm{T}(\mathrm{u} \wedge \overline{\mathrm{V}}))(\mathrm{x})
$$

for any strategy $\sigma$ of $I$ and any stop rule $t \geq 1$. Next, for each $y \in X$, choose a strategy $\bar{\tau}(y)$ for II such that for all strategies $\sigma$ of I,

$$
E_{y, \sigma, \bar{\tau}(y)}\left(u^{*}\right)<\bar{V}(y)+\frac{\varepsilon}{2} .
$$

The existence of $\tau^{1}$ follows from Theorem 4.8 and that of $\bar{\tau}(y)$ from the definition of $\bar{V}(y)$.

Let

$$
\bar{t}(h)=\inf \left\{k \geq 1: u\left(x_{k}\right)>\bar{v}\left(x_{k}\right)\right\}
$$

where $\inf (\phi)=\infty$. Plainly, $\bar{t}$ is a stopping time. Now define $\tau$ as follows:

$$
\tau_{0}=\tau_{0}^{1}
$$


$\tau_{n}\left(z_{1}, z_{2}, \ldots, z_{n}\right)=\tau_{n}^{1}\left(z_{1}, z_{2}, \ldots, z_{n}\right)$ if $1 \leq n<\bar{t}(h)$,

$$
=\bar{\tau}\left(x_{i n-\bar{t}}\left(z_{\bar{t}+1}, z_{\bar{t}+2}, \ldots, z_{n}\right) \text { if } \bar{t}(h) \leq n,\right.
$$

where $h=\left(z_{1}, z_{2}, \ldots, z_{n}, \ldots\right)$.

Fix a strategy $\sigma$ for player I. We will show that there is a stop rule $s \geq 1$ such that for all stop rules $t \geq s$,

$$
E_{x, \sigma, \tau}\left(u\left(x_{l}\right)\right) \leq(T(u \wedge \bar{v}))(x)+\varepsilon
$$

It follows from (5.7) and Lemma 5.1 that

$$
E_{\sigma, \tau}\left(u^{*}\right) \leq(T(u \wedge \bar{V}))(x)+\varepsilon
$$

Since $\sigma$ is arbitrary, this implies that

$$
\overline{\mathrm{V}}(\mathrm{x}) \leq(\mathrm{T}(\mathrm{u} \wedge \overline{\mathrm{V}}))(\mathrm{x})+\varepsilon
$$

As $\varepsilon$ is arbitrary, this will complete the proof of the theorem, once we have established (5.7).

To prove (5.7), use [11, Lemma 1] to choose a stop rule $\bar{s} \geq 1$ such that

$$
P_{\sigma, \tau}(\{\bar{t}<\infty\}) \leq P_{\sigma, \tau}(\{\bar{t} \leq \bar{s}\})+\frac{\varepsilon}{4(\|u\|+1)} .
$$

Use Lemma 5.1 and (5.6) to choose, for each $p=\left(z_{1}, z_{2}, \ldots, z_{n}\right)$, a stop rule $\bar{r}(p) \geq 1$ such that

$$
\mathrm{E}_{x_{n}, \sigma[p], \bar{\tau}\left(x_{n}\right)}\left(u\left(x_{t}\right)\right) \leq \bar{V}\left(x_{n}\right)+\frac{\varepsilon}{2}
$$

for all stop rules $t \geq \bar{r}(p)$. 
Define a stop rule $s$ as follows:

$$
\begin{aligned}
s(h) & =\bar{t}(h)+\bar{r}\left(p_{\bar{i}}(h)\right)\left(\dot{z}_{\bar{i}+1}, z_{\bar{t}+2}, \ldots\right) \text { if } \bar{t}(h) \leq \bar{s}(h), \\
& =\bar{s}(h) \text { if } \bar{t}(h)>\bar{s}(h),
\end{aligned}
$$

where $h=\left(z_{1}, z_{2}, \ldots\right)$.

Let $t$ be a stop rule such that $t \geq s$. Condition on $p_{u \bar{t}}$ and calculate as follows:

$$
\begin{aligned}
& E_{x, \sigma, \tau}\left(u\left(x_{t}\right)\right) \\
& =\int_{[\bar{i} \leq t)} E_{x_{\bar{t}}, \sigma\left[p_{\bar{t}}\right], \bar{\tau}\left(x_{\hat{t}}\right)}\left(u\left(x_{t\left[p_{\bar{t}}\right]}\right)\right) d P_{\sigma, \tau} \\
& +\int_{[\bar{D} l t]} u\left(x_{l}\right) d P_{\sigma, \tau} \\
& \leq \int_{(\overline{\mathrm{i}} \leq \bar{s})} \mathrm{E}_{\mathrm{x}_{\overline{\mathrm{i}}} \sigma\left[\mathrm{p}_{\overline{\mathrm{i}}}\right], \bar{\tau}\left(\mathrm{x}_{\overline{\mathrm{i}}}\right)}\left(\mathrm{u}\left(\mathrm{x}_{\mathrm{t}\left[\mathrm{p}_{\overline{\mathrm{i}}}\right]}\right)\right) \mathrm{dP} \mathrm{P}_{\sigma, \tau}+\frac{\varepsilon}{4} \\
& +\int_{\{\bar{D} l\}} u\left(x_{t}\right) d P_{\sigma, \tau} \\
& \leq \int_{[\bar{i} \leq s)} \bar{V}\left(x_{\bar{l}}\right) d P_{\sigma, \tau}+\frac{3 \varepsilon}{4}+\int_{(\bar{D})\}} u\left(x_{t}\right) d P_{\sigma, \tau} \\
& \leq \int_{\{\bar{L}<t]} \bar{V}\left(x_{i}\right) d P_{\sigma, \tau}+\varepsilon+\int_{[\bar{D} t\}} u\left(x_{t}\right) d P_{\sigma, \tau} \\
& =\int(u \wedge \bar{V})\left(x_{\bar{i} \sim}\right) d P_{\sigma, \tau}+\varepsilon \\
& =\int(\mathrm{u} \wedge \bar{V})\left(\mathrm{x}_{\bar{u} \iota_{\mathrm{\imath}}}\right) \mathrm{dP}{ }_{\sigma, \tau^{1}}+\varepsilon \\
& \leq(\mathrm{T}(\mathrm{u} \wedge \overline{\mathrm{V}}))(\mathrm{x})+\varepsilon,
\end{aligned}
$$


where the first inequality uses (5.8) and the set inclusions $\{\overline{\mathrm{t}} \leq \overline{\mathrm{s}}\} \subseteq\{\overline{\mathrm{t}} \leq \mathrm{t}\} \subseteq\{\overline{\mathrm{t}}<\infty\}$, the second inequality is by virtue of (5.9) and the fact that on $\{\bar{i} \leq \bar{s}\}, t\left[p_{\hat{t}}\right] \geq s\left[p_{\hat{t}}\right]=\bar{r}\left(p_{\hat{t}}\right)$, and the last inequality is by virtue of (5.5).

\section{Borel stochastic games}

In [7], we studied stochastic games in a Borel measurable setting. More precisely, we made the following assumptions:

(i) $\mathrm{X}$ and $\mathrm{A}$ are Borel subsets of Polish spaces.

(ii) $\mathrm{B}$ is a compact metric space.

(iii) $q$ is a Borel measurable transition function, that is, for each $(x, a, b) \in X \times A \times B$, $q(\cdot \mid x, a, b)$ is a countably additive probability measure on the Borel $\sigma$-field of $X$, and for each Borel subset $E$ of $X, q(E \mid \bullet, \bullet, \bullet)$ is Borel measurable on $X \times A \times B$.

(iv) For every fixed Borel subset $E$ of $X, x \in X$ and $a \in A, q(E \mid x, a, \bullet)$ is continuous on $B$.

(v) $u$ is a bounded, upper analytic function on $X$, that is, for every real $c$, the set $\{u>c\}$ is analytic.

Furthermore, both players are required to use measurable strategies. Recall that a measurable strategy $\sigma$ for player $I$ is a sequence $\sigma_{0}, \sigma_{1}, \ldots$, where $\sigma_{0} \in \mathrm{P}_{\mathrm{c}}(\mathrm{A})$, the set of countably additive probability measures on the Borel subsets of $A$, and, for $n \geq 1, \sigma_{n}$ is a universally measurable mapping from $Z^{n}$ to $P_{c}(A)$ (that is, measurable when $Z^{n}$ is endowed with its $\sigma$-field of universally measurable subsets and $P_{c}(A)$ is equipped with the Borel $\sigma$ field generated by the weak topology on $\mathrm{P}_{c}(A)$ ). A measurable strategy $\tau$ for player II is defined analogously.

Now equip $A, B$ and $X$ with the topologies with respect to which they are Borel subsets of Polish spaces and let $Z=A \times B \times X$ have the resulting product topology. Finally, give $\mathrm{H}=\mathrm{Z}^{\mathrm{N}}$ the product of copies of this topology on $\mathrm{Z}$ and let $\tilde{\mathrm{C}}_{\mathrm{H}}$ be the Borel $\sigma$-field generated by this product topology on $\mathrm{H}$. 
Measurable strategies $\sigma$ and $\tau$, together with an initial state $x$, induce via the Ionescu Tulcea theorem a countably additive probability measure $\widetilde{P}_{x, \sigma, \tau}$ on $\widetilde{R}_{\mathrm{H}}$. The expected payoff to $I$ is then $\int_{u^{*}} d \widetilde{P}_{x, \sigma, \tau}$. Let us denote this game by $n_{c}(x)$. It was proved in [7] that $n_{c}(x)$ has a value for each initial state $x$.

Suppose now that we fix, for each triple $(\mathrm{x}, \mathrm{a}, \mathrm{b}) \in \mathrm{X} \times \mathrm{A} \times \mathrm{B}$, a finitely additive extension of $q(\cdot \mid x, a, b)$ to the power-set of $X$. Denote the extension by the same symbol $\mathrm{q}(\cdot \mid \mathrm{x}, \mathrm{a}, \mathrm{b})$. Then, according to Theorem 2.1 , the game $n(\mathrm{u})(\mathrm{x})$ has a value for every $\mathrm{x} \in$ $\mathrm{X}$. A natural question then is whether the values of $n(\mathrm{u})(\mathrm{x})$ and $n_{\mathrm{c}}(\mathrm{u})(\mathrm{x})$ coincide for every $x \in X$. If these values are the same, then one can ask if the players have good measurable strategies which work against all strategies of the opponent, measurable or nonmeasurable. The rest of the paper is devoted to answering both questions in the affirmative under conditions (i), (ii), (iii), ( $v$ ) above and the following strengthening of (iv):

(iv') For every fixed Borel subset $E$ of $X$ and $x \in X$, the family $(q(E \mid x, a, \bullet): a \in A)$ is equicontinuous on $B$.

\section{One-shot games}

As a first step towards the solution of the problems posed in the previous section, we study one-shot games and prove that, under appropriate conditions, the values of such games remain the same whether the players use only countably additive mixed strategies or use finitely additive ones. The key to the proof of this result is a "Fubini" - type theorem for finitely additive probability measures.

Theorem 7.1. Let $(\Omega, Q)$ be a measurable space, let $Y$ be a compact metric space and denote by ${ }_{Y}$ the Borel $\sigma$-field of $Y$. Suppose that $\tilde{\mathrm{f}}$ is a bounded, real-valued function on $\Omega \times Y$ such that $\{f(\omega, \bullet): \omega \in \Omega\}$ is equicontinuous on $Y$ and $\tilde{f}(\bullet, y)$ is $Q$-measurable for every $y \in Y$. Let $\mu, v$ be finitely additive probability measures on $Q, \alpha_{Y}$, respectively. Then

$$
\iint \tilde{f}(\omega, y) d \mu(\omega) d v(y)=\iint f(\omega, y) d v(y) d \mu(\omega) .
$$

Sketch of proof. First, note that the iterated integrals above are meaningful. Indeed, the map $y \rightarrow \int \tilde{f}(\omega, y) d \mu(\omega)$ is continuous on $Y$, as can be seen by using the equicontinuity of 
$\{\tilde{f}(\omega, \bullet): \omega \in \Omega$. Next, use the Riesz representation theorem to get a countably additive probability measure $v^{*}$ on $k_{Y}$ such that

$$
\int g d v=\int g d v^{*}
$$

for every real-valued, continuous function $g$ on $Y$. Hence, the map $\omega \rightarrow \int \tilde{f}(\omega, y) d v(y)=$ $\int \tilde{f}(\omega, y) d v^{*}(y)$ is $Q$-measurable on $\Omega$. In order to prove the equality of the iterated integrals, use the hypotheses to prove that for $\varepsilon>0$, there is a finite $Q$-measurable partition $\left\{\Omega_{1}, \Omega_{2}, \ldots, \Omega_{m}\right\}$ of $\Omega$ and a finite $\Omega_{Y}$ - measurable partition $\left\{Y_{1}, Y_{2}, \ldots, Y_{n}\right\}$ of $Y$ such that if $(\omega, y)$ and $\left(\omega^{\prime}, y^{\prime}\right)$ both belong to $\Omega_{\mathrm{i}} \times Y_{\mathrm{j}}$, then

$$
\left|\tilde{f}(\omega, y)-\widetilde{f}\left(\omega^{\prime}, y^{\prime}\right)\right|<\varepsilon . \square
$$

The result on one-shot games follows.

Theorem 7.2. Let $(\Omega, Q)$ be a measurable space, let $Y$ be a compact metric space with Borel $\sigma$-field $t_{Y}$. Let $g$ be a bounded, real-valued function on $\Omega \times Y$ such that $\{g(\omega, \bullet): \omega \in \Omega\}$ is equicontinuous on $\mathrm{Y}$ and $\mathrm{g}(\bullet, y)$ is $Q$-measurable for every $\mathrm{y} \in \mathrm{Y}$. Then there is a real number $v$ such that

(a) for every $\varepsilon>0$, there is a countably additive probability measure $\mu^{*}$ on $Q$ such that for all finitely additive probability measures $v$ on ${ }_{Y}$

$$
\iint g(\omega, y) d \mu^{*}(\omega) d v(y)=\iint g(\omega, y) d v(y) d \mu^{*}(\omega) \geq v-\varepsilon,
$$

(b) there is a countably additive probability measure $v^{*}$ on $\beta_{Y}$ such that for all finitely additive probability measures $\mu$ on $Q$

$$
\iint g(\omega, y) d \mu(\omega) d v^{*}(y)=\iint g(\omega, y) d v^{*}(y) d \mu(\omega) \leq v .
$$

Proof. Use Theorem 2 from Ky Fan [4] and Theorem 7.1.

Theorem 7.2 states that the one-shot game with payoff $g(\omega, y)$ has the same value whether the players play mixed strategies which are finitely additive or use only countably additive mixed strategies. Moreover, the order of integration in evaluating the payoff is 
irrelevant, even when finitely additive mixed strategies are used. Finally, both players have good strategies which are countably additive.

From this point onwards, we will assume that conditions (i), (ii), (iii), (iv') and (v) are in force for the rest of the paper. Furthermore, for each countably additive probability measure on the Borel $\sigma$-field of $X(A)(B)$, we fix a finitely additive extension to the powerset of $X(A)(B)$.

We now consider the games $Q(\varphi)(x), x \in X$, when $\varphi$ is a bounded, upper analytic function on $X$. These games were introduced with arbitrary $\varphi$ in section 4 . Recall that $(S \varphi)(x)$ is the value of the game $Q(\varphi)(x)$ when both players use finitely additive mixed strategies.

Theorem 7.3. Let $\varphi$ be a bounded, upper analytic function on $X$. Then $S \varphi$ is an upper analytic function. For every $\varepsilon>0$, there is a universally measurable function $f: X \rightarrow P_{c}(A)$ such that for every $\mathrm{x} \in \mathrm{X}$

$$
\inf _{v \in P(B)} \iiint \varphi\left(x_{1}\right) q\left(d x_{1} \mid x, a, b\right) f(x)(d a) v(d b) \geq(S \varphi)(x)-\varepsilon
$$

Moreover, there is a universally measurable function $\mathrm{g}: \mathrm{X} \rightarrow \mathrm{P}_{c}(\mathrm{~B})$ such that for every $\mathrm{x} \in \mathrm{X}$

$$
\sup _{\mu \in P(A)} \iiint \varphi\left(x_{1}\right) q\left(d x_{1} \mid x, a, b\right) \mu(d a) g(x)(d b) \leq(S \varphi)(x) .
$$

Proof. According to a result of Nowak [8, Theorem 5.1], there is an upper analytic function $V$ on $X$, a universally measurable function $\mathrm{g}: X \rightarrow P_{c}(B)$ and, given $\varepsilon>0$, a universally measurable function $\mathrm{f}: \mathrm{X} \rightarrow \mathrm{P}_{\mathrm{c}}(\mathrm{A})$ such that

$$
\int \varphi\left(x_{1}\right) q\left(d x_{1} \mid x, a, b\right) f(x)(d a) \geq V(x)-\varepsilon
$$

for all $b \in B$ and all $x \in X$, and

$$
\int \varphi\left(x_{1}\right) q\left(d x_{1} \mid x, a, b\right) g(x)(d b) \leq V(x)
$$

for all $a \in A$ and all $x \in X$. 
If we now integrate (7.3) with respect to any $v \in P(B)$, we get (7.1) with $(S \varphi)(x)$ replaced by $V(x)$. On the other hand, integrate (7.4) with respect to any $\mu \in P(A)$ and interchange the order of integration to get (7.2), again with $(S \varphi)(x)$ replaced by $V(x)$. To justify interchange of the order of integration, apply Theorem 7.1 with $\Omega=A, Q=$ the $\sigma$ field on A generated by the analytic subsets of $A, Y=B$ and

$$
\widetilde{f}(a, b)=\int \varphi\left(x_{1}\right) q\left(d x_{1} \mid x, a, b\right), a \in A, b \in B .
$$

That $\tilde{f}$ satisfies the equicontinuity condition in Theorem 7.1 follows from condition (iv') of section 6 by standard arguments.

The proof is concluded by observing now that V must be $S \varphi$.

\section{Leavable games}

We now consider the "leavable" games $\check{L}(\varphi)(x)$ and $\swarrow^{*}(\varphi)(x), x \in X$, when $\varphi$ is a bounded, upper analytic function on $X$. These games with arbitrary $\varphi$ were studied in section 4.

Suppose $\sigma, \tau$ are measurable strategies for players I and II, respectively. For fixed $\mathbf{x} \in \mathbf{X}, \sigma$ and $\tau$ together determine a countably additive probability measure $\widetilde{P}_{\mathbf{x}, \sigma, \tau}$ on the $\sigma$ field $\tilde{\Omega}_{H}$ via the Ionescu Tulcea theorem, as was mentioned in section 6 . On the other hand, since all countably additive probability measures on $\mathrm{X}, \mathrm{A}, \mathrm{B}$, have been extended to the respective power-sets, $\sigma, \tau$ also qualify as strategies in the sense of section 2 . Hence, as described in section $2, \sigma, \tau$ induce a finitely additive probability measure $P_{x, \sigma, \tau}$ on the Borel $\sigma$-field $\varangle_{\mathrm{H}}$, generated by the product of copies of the discrete topology on $\mathrm{Z}$. It was

proved in [10, Theorem 6.1] that $\tilde{P}_{x, \sigma, \tau}=P_{x, \sigma, \tau}$ on $\tilde{\beta}_{H}$. In the sequel, therefore, we will write $P_{x, \sigma, \tau}$ for $\tilde{P}_{x, \sigma, \tau}$.

We remind the reader that conditions (i), (ii), (iii), (iv') and (v) of section 6 are in force.

Lemma 8.1. For every $n=0,1,2, \ldots$, the function $U_{n}$ is upper analytic. Moreover, $U=$ $\sup _{n} U_{n}$ and, consequently, $U$ is upper analytic. [The functions $U_{n}$ and $U$ are defined in section 4.]

Proof. The first assertion is proved by induction on $\mathrm{n}$ by using the fact noted in Theorem 7.3 that the operator $S$ preserves bounded, upper analytic functions on $X$. For the second 
assertion, note that $\sup _{n} U_{n} \geq u$ and, by [7, Lemma 4.1], $\sup _{n} U_{n} \geq S\left(\sup _{n} U_{n}\right)$. Hence, $\mathrm{U}=\sup _{\mathrm{n}} \mathrm{U}_{\mathrm{n}}$ by virtue of Lemma 4.2.

We say that $(\sigma(x))_{x \in X}$ is a measurable family of strategies for player I if for every $x \in X, \sigma(x)$ is a measurable strategy for $I$ and, for every $n \geq 0,(\sigma(x))_{n}\left(z_{1}, z_{2}, \ldots, z_{n}\right)$ is a universally measurable mapping from $X \times Z^{n}$ to $P_{c}(A)$. One defines a measurable family of strategies for player II analogously. A stopping time (stop rule) $t$ is measurable if, for every $n \geq 0$, the set $\{t \leq n\}$ is a universally measurable subset of $H$. A policy for player I is a pair $(\sigma, t)$, where $\sigma$ is a strategy for I and t a stop rule. A policy $(\sigma, t)$ for $I$ is measurable if $\sigma$ is a measurable strategy for $I$ and $t$ is a measurable stop rule. We say that $(\sigma(x), t(x))_{x \in X}$ is a measurable family of policies for player I if $(\sigma(x))_{x \in X}$ is a measurable family of strategies for $I, t(x)$ is a stop rule for every $x \in X$ and $t(x)(h)$ is a universally measurable function on $\mathrm{X} \times \mathrm{H}$.

Lemma 8.2. For every $n \geq 0$ and $\varepsilon>0$, player I has a measurable family of policies $\left(\sigma_{\varepsilon}^{n}(x), t^{n}(x)\right)_{x \in X}$ such that $t^{n}(x) \leq n, x \in X$, and such that for any strategy (measurable or not) $\tau$ of II,

$$
E_{x, \sigma_{\varepsilon}^{n}(x), \tau}\left(u\left(x_{\imath^{n}(x)}\right)\right) \geq U_{n}(x)-\varepsilon
$$

for every $x \in X$.

Proof. The proof is by induction on $\mathrm{n}$ and uses Theorem 7.3 and Lemma 8.1. We omit the details as they are easily supplied by examining the proof of [7, Lemma 4.2] and Lemma 4.4.

Lemma 8.3. For every $\varepsilon>0$, player I has a measurable family of policies $\left(\sigma_{\varepsilon}(x), t_{\varepsilon}(x)\right)_{x \in X}$ such that for any strategy (measurable or not) $\tau$ of player II

$$
E_{x, \sigma_{\varepsilon}(x), \tau}\left(u\left(x_{t_{\varepsilon}(x)}\right)\right) \geq U(x)-\varepsilon
$$

for every $x \in X$. Furthermore, player II has a measurable family of strategies $(\tau(x))_{x \in X}$ such that for any policy (measurable or not) $(\sigma, t)$ of player I 


$$
E_{x, \sigma, \tau(x)}\left(u\left(x_{1}\right)\right) \leq U(x)
$$

for every $x \in X$.

Proof. For $x \in X$, define $n(x)$ to be the least $k \geq 0$ such that $U_{k}(x)>U(x)-\varepsilon / 2$. Use Lemma 8.1 to see that $n(x)$ is a universally measurable function of $x$. For each $m \geq 0$, choose a measurable family of policies $\left(\sigma_{\varepsilon / 2}^{\mathrm{m}}(\mathrm{x}), \mathrm{t}^{\mathrm{m}}(\mathrm{x})\right)_{x \in X}$ for player I such that $(8.1)$ is satisfied with $\varepsilon$ replaced by $\varepsilon / 2$. Set

$$
\begin{aligned}
& \sigma_{\varepsilon}(x)=\sigma_{\varepsilon / 2}^{n(x)}(x) \\
& t_{\varepsilon}(x)=t^{n(x)}(x) .
\end{aligned}
$$

It is straightforward to verify that $\left(\sigma_{\varepsilon}(x), t_{\varepsilon}(x)\right)_{x \in X}$ satisfies the assertion of the lemma.

For the second assertion, use Theorem 7.3 to fix a universally measurable function $\mathrm{g}: \mathrm{X} \rightarrow \mathrm{P}_{\mathrm{c}}(\mathrm{B})$ such that

$$
\sup _{\mu \in P(A)} \iiint U\left(x_{1}\right) q\left(d x_{1} \mid x, a, b\right) \mu(d a) g(x)(d b) \leq(S U)(x) .
$$

for every $x \in X$. Let

$$
\begin{aligned}
& \tau(x)_{0}=g(x) \\
& \tau(x)_{n}\left(z_{1}, z_{2}, \ldots, z_{n}\right)=g\left(x_{n}\right), n \geq 1 .
\end{aligned}
$$

Plainly, $(\tau(x))_{x \in X}$ is a measurable family of strategies for II. It is now easy to verify (8.2) by induction on $j(t)$ exactly as in the proof of Lemma 4.6.

Theorem 8.4. For every $\varepsilon>0$, player I has a measurable family of policies $\left(\sigma_{\varepsilon}(x), t_{\varepsilon}(x)\right)_{x \in X}$, with $t_{\varepsilon}(x) \geq 1$ for every $x \in X$, such that for any strategy (measurable or not) $\tau$ of player II

$$
E_{\sigma_{\varepsilon}(x), \tau}\left(u\left(x_{\varepsilon_{\varepsilon}(x)}\right)\right) \geq(S U)(x)-\varepsilon
$$


for every $x \in X$. Moreover, player $\Pi$ has a measurable family of strategies $(\tau(x))_{x \in X}$ such that for any policy (measurable or not) $(\sigma, t)$ of player $I$, with $t \geq 1$,

$$
E_{x, \sigma, \tau(x)}\left(u\left(x_{t}\right)\right) \leq(S U)(x)
$$

for every $x \in X$.

Proof. The proof is exactly like that of Theorem 4.8, except that we use Lemma 8.3 instead of Lemmas 4.5 and 4.6.

Theorem 8.4 implies that the game $2^{*}(\varphi)(x)$ has the same value whether the players use measurable strategies or nonmeasurable ones. Furthermore, both players have good strategies which are measurable. Another consequence of Theorem 8.4 is the following.

Corollary 8.5. The operator $T$ maps bounded, upper analytic functions on $\mathrm{X}$ to bounded, upper analytic functions on $\mathrm{X}$.

We remind the reader that for an arbitrary bounded function $w$ on $X,(T w)(x)$ was defined in section 4 to be the value of the game $2^{*}(w)(x)$ for $x \in X$.

We now define, as in [7], functions $Q_{\xi}, \xi<\omega_{1}$, on $X$ by transfinite induction as follows:

$$
\mathrm{Q}_{0}=\mathrm{Tu}
$$

and, for $\xi>0$,

$$
Q_{\xi}=T\left(u \wedge \inf _{\eta<\xi} Q_{\eta}\right)
$$

Set

$$
Q=\inf _{\xi<\omega_{1}} Q_{\xi}
$$

The main properties of the functions above were established in [7] and are summarized below. 
Theorem 8.6. (a) The functions $Q_{\xi}, \xi<\omega_{1}$, and $Q$ are upper analytic.

(b) $\mathrm{T}(\mathrm{u} \wedge \mathrm{Q})=\mathrm{Q}$.

\section{Nonleavable games}

We are now in a position to settle the questions posed in section 6 regarding the games $n(\mathrm{u})(\mathrm{x})$ and $n_{\mathrm{c}}(\mathrm{u})(\mathrm{x})$.

Theorem 9.1. For every $\varepsilon>0$, player I has a measurable family of strategies $(\sigma(x))_{x \in X}$ such that for any strategy (measurable or not) $\tau$,

$$
E_{x, \sigma(x), \tau}\left(u^{*}\right) \geq Q(x)-\varepsilon
$$

for every $x \in X$.

Proof. We consider the games $2^{*}(u \wedge Q)(x), x \in X$. According to Theorem 8.6, $(T(u \wedge Q))(x)=Q(x), x \in X$. So we can use Theorem 8.4 to choose for each $\delta>0$ a measurable family of policies $(\bar{\sigma}(x, \delta), \bar{t}(x, \delta))_{x \in X}$, with $\bar{i}(x, \delta) \geq 1$ for every $x \in X$, such that for every strategy (measurable or not) $\tau$ of II

$$
E_{x, \bar{\sigma}(x, \delta), \tau}(u(x), \delta(x, \delta)) \geq Q(x)-\varepsilon
$$

for every $x \in X$.

Let $\delta_{0}, \delta_{1}, \ldots$ be positive numbers such that $\sum_{n} \delta_{n}<\varepsilon$. For each $x \in X$ and $n \geq 0$, set $\sigma^{n}(x)=\bar{\sigma}\left(x, \delta_{n}\right), t_{n}(x)=\bar{t}\left(x, \delta_{n}\right)$. Let $\sigma(x)$ be the sequential composition of $\left(\sigma^{n}, t_{n}\right), n \geq 0$, at $x$ (see section 5 for the definition of sequential composition). Since the family of policies $\left(\sigma^{n}(x), t_{n}(x)\right)_{x \in X}, n \geq 0$, are measurable, it follows that $(\sigma(x))_{x \in X}$ is a measurable family of strategies.

To prove (9.1), just imitate the proof of Theorem 5.2, replacing the function $\varphi$ by Q.

Theorem 9.2. For every $\varepsilon>0$ and $\xi<\omega_{1}$, player II has a measurable family of strategies $\left(\tau^{\xi}, \varepsilon(x)\right)_{X \in X}$ such that for any strategy (measurable or not) $\sigma$ of player I

$$
E_{x, \sigma, \tau, \xi(x)}\left(u^{*}\right) \leq Q_{\xi}(x)+\varepsilon
$$


for every $x \in X$.

Proof. The proof is by induction on $\xi$. Consider the case when $\xi=0$. Use Theorem 8.4 to choose a measurable family of strategies $(\bar{\tau}(\mathrm{X}))_{\mathrm{X} \in \mathrm{X}}$, which is optimal for II in the games $Z^{*}(u)(x), x \in X$, that is, for any policy $(\sigma, t)$ of $I$, with $t \geq 1$,

$$
E_{x, \sigma, \bar{\tau}(x)}\left(u\left(x_{l}\right)\right) \leq Q_{0}(x)
$$

for every $x \in X$.

Set

$$
\tau^{0, \varepsilon}(x)=\bar{\tau}(x), x \in X
$$

Fix $x \in X$ and let $\sigma$ be a strategy for $I$. Then

$$
\begin{aligned}
E_{x, \sigma, \tau^{0, \varepsilon}(x)}\left(u^{*}\right) & =E_{x, \sigma, \bar{\tau}(x)}\left(u^{*}\right) \\
& \leq \sup _{l \geq 1} E_{x, \sigma, \bar{\tau}(x)}\left(u\left(x_{t}\right)\right) \\
& \leq Q_{0}(x),
\end{aligned}
$$

where the first inequality is by virtue of Lemma 5.1 and the second by virtue of (9.3).

For the inductive step, let $\xi>0$ and assume that the result is true for all $\eta<\xi$. Use Theorem 8.4 once again to choose a measurable family $(\bar{\tau}(x))_{x \in X}$ of optimal strategies for $\Pi$ in the games $Z^{*}\left(u \wedge \inf _{\eta<\xi} Q_{\eta}\right)(x), x \in X$. Let

$$
\bar{t}(h)=\inf \left\{k \geq 1: u\left(x_{k}\right)>\left(\inf _{\eta<\xi} Q_{\eta}\right)\left(x_{k}\right)\right\}
$$

where $\inf (\phi)=\infty$.

Then $\bar{t}$ is a measurable stopping time.

For $\eta<\xi$, let 


$$
\left.C_{\eta}=\left\{x \in X: Q_{\eta}(x)<\inf _{\zeta<\xi} Q_{\zeta}\right)(x)+\varepsilon / 4\right\}
$$

Clearly, the sets $C_{\eta}$ are universally measurable. Define $\tau^{\xi, \varepsilon}$ as follows:

$$
\tau^{\xi, \varepsilon}(x)_{0}=\bar{\tau}(x)_{0}
$$

and, for $\mathrm{n} \geq 1$,

$$
\begin{aligned}
\tau^{\xi, \varepsilon}(x)_{n}\left(z_{1}, z_{2}, \ldots, z_{n}\right)= & \bar{\tau}(x)_{n}\left(z_{1}, z_{2}, \ldots, z_{n}\right), \text { if } n<\bar{t}(h), \\
& =\tau^{\eta, \varepsilon / 4}\left(x_{\bar{t}}\right)_{n-\bar{t}}\left(z_{\bar{t}+1}, z_{\bar{t}+2}, \ldots, z_{n}\right), \\
& \text { if } n \geq \bar{t}(h) \& x_{\bar{t}} \in C_{\eta}-U_{\eta^{\prime}<\eta} C_{\eta^{\prime}},
\end{aligned}
$$

where $h=\left(z_{1}, z_{2}, \ldots\right)$.

Clearly, $\left(\tau^{\xi}, \varepsilon(x)\right)_{x \in X}$ is a measurable family of strategies.

Fix $x \in X$ and let $\sigma$ be a strategy for player I. To prove (9.2), just repeat the proof of Theorem 5.3 with $\inf _{\eta<\xi} Q_{\eta}$ playing the role of $\bar{V}$.

Corollary 9.3. Let $\varepsilon>0$. Player I has a measurable family of strategies $(\sigma(x))_{x \in X}$ such that for every strategy (measurable or not) $\tau$ of $\Pi$

$$
E_{x, \sigma(x), \tau}\left(u^{*}\right) \geq Q(x)-\varepsilon
$$

for every $x \in X$. Player II has a measurable family of strategies $(\tau(x))_{x \in X}$ such that for every strategy (measurable or not) $\sigma$ of I

$$
E_{x, \sigma, \tau(x)}\left(u^{*}\right) \leq Q(x)+\varepsilon
$$

for every $x \in X$.

Proof. The first assertion is just Theorem 9.1. For the second assertion, let

$$
C_{\xi}=\left\{x \in X: Q_{\xi}(x)=Q(x)\right\}, \xi<\omega_{1}
$$


Define

$$
\tau(x)=\tau^{\xi, \varepsilon}(x) \text { if } x \in C_{\xi}-U_{\eta<\xi} C_{\eta} \text {, }
$$

where $\tau^{\xi, \varepsilon}$ is defined in Theorem 9.2. It is proved in [7, Corollary 6.5] that $(\tau(x))_{x \in X}$ is a measurable family of strategies.

Finally, fix $x \in X$ and let $\sigma$ be a strategy for I. If $x \in C_{\xi}-U_{\eta<\xi} C_{\eta}$ for $\xi<\omega_{1}$, then

$$
\begin{aligned}
E_{x, \sigma, \tau(x)}\left(u^{*}\right)= & E_{x, \sigma, \tau \xi, \varepsilon(x)}\left(u^{*}\right) \\
& \leq Q_{\xi}(x)+\varepsilon \\
& =Q(x)+\varepsilon,
\end{aligned}
$$

where the inequality is by virtue of Theorem 9.2.

Corollary 9.3 states that the games $n(u)(x)$ and $n_{c}(u)(x)$ have the same value $Q(x)$. Moreover, both players have good measurable strategies in $n(u)(x), x \in X$. Hence, the value function $Q$ can be calculated entirely in terms of measurable strategies, so that the finitely additive extensions fixed for countably additive measures in section 7 are irrelevant insofar as the value is concerned. 


\section{References}

1. L.E. Dubins and L.J. Savage, Inequalities for Stochastic Processes, Dover, New York (1976).

2. L.E. Dubins, On Lebesgue-like extensions of finitely additive measures, Ann. Probab. 2, (1974), 456-463.

3. L. Dubins, A. Maitra, R. Purves and W. Sudderth, Measurable, nonleavable gambling problems, Israel J. Math. 67 (1989), 257-271.

4. Ky Fan, Minimax theorems, Proc. Nat. Acad. Sci. U.S.A. 39 (1953), 42-47.

5. D. Heath and W. Sudderth, On a theorem of de Finetti, oddsmaking and game theory, Ann. Math. Statist. 43 (1972), 2072-2077.

6. A. Maitra and W. Sudderth, An operator solution of stochastic games, preprint (1990).

7. A. Maitra and W. Sudderth, Borel stochastic games with limsup payoff, Ann. Probab., to appear.

8. A.S. Nowak, Universally measurable strategies in zero-sum stochastic games, Ann. Probab. 13 (1985), 269-287.

9. R. Purves and W. Sudderth, Some finitely additive probability, University of Minnesota Technical Report No. 220 (1973).

10. R. Purves and W. Sudderth, Some finitely additive probability, Ann. Probab. 4 (1976), 259-276.

11. R. Purves and W. Sudderth, How to stay in a set or König's Lemma for random paths, Israel J. Math. 43 (1982), 139-153.

12. W. Sudderth, A "Fatou equation" for randomly stopped variables, Ann. Math. Statist. 42 (1971), 2143-2146. 